\title{
NOMINAL LHC BEAM INSTABILITY OBSERVATIONS IN THE CERN PROTON SYNCHROTRON
}

\author{
R. Steerenberg, G. Arduini, E. Benedetto, A. Blas, W. Höfle, E. Métral, M. Morvillo, C. Rossi, \\ G. Rumolo \\ CERN, Geneva, Switzerland
}

\begin{abstract}
The nominal LHC beam has been produced successfully in the CERN Proton Synchrotron since 2003. However, after having restarted the CERN PS in spring 2006, the LHC beam was set-up and observed to be unstable on the $26 \mathrm{GeV} / \mathrm{c}$ extraction flat top. An intensive measurement campaign was made to understand the instability and to trace its source. This paper presents the observations, possible explanations and the necessary measures to be taken in order to avoid this instability in the future.
\end{abstract}

CERN, 


\title{
NOMINAL LHC BEAM INSTABILITY OBSERVATIONS IN THE CERN PROTON SYNCHROTRON
}

\author{
R. Steerenberg, G. Arduini, E. Benedetto, A. Blas, W. Höfle, E. Métral, M. Morvillo, C. Rossi, \\ G. Rumolo, CERN, Geneva, Switzerland.
}

\section{Abstract}

The nominal LHC beam has been produced successfully in the CERN Proton Synchrotron since 2003. However, after having restarted the CERN PS in spring 2006, the LHC beam was set-up and observed to be unstable on the $26 \mathrm{GeV} / \mathrm{c}$ extraction flat top. An intensive measurement campaign was made to understand the instability and to trace its source. This paper presents the observations, possible explanations and the necessary measures to be taken in order to avoid this instability in the future.

\section{INTRODUCTION}

In 2001 electron cloud build-up was observed on the beam position monitors in the PS, on the extraction flat top and in the PS to SPS transfer line [1]. Electron multipacting occurred in the last few milliseconds before extraction, too short for the beam to become unstable. During the following years the beam was continuously improved with the emphasis on the reproducibility from bunch to bunch and from shot to shot. For this reason a longitudinal coupled bunch mode feedback was installed in 2004, resulting in a reduced bunch length spread along the bunch train. At the same time a transverse instability, during the last part of the acceleration, was observed. At the restart in 2006, after an 18 month shut down, the beam was observed to be unstable again, but disabling the coupled bunch mode feedback did not revert the situation.

For that reason a measurement campaign was started on the nominal LHC $25 \mathrm{~ns}$ beam, with an extracted bunch intensity of $1.3 \mathrm{E} 10^{11}$.

\section{FLAT TOP RF MANIPULATIONS}

18 bunches are accelerated to $26 \mathrm{GeV} / \mathrm{c}$ using a harmonic $21 \mathrm{RF}$ system. On the flat top the 18 bunches undergo a double-splitting twice to obtain 72 bunches in harmonic 84 buckets creating a $25 \mathrm{~ns}$ bunch spacing. At the end of the adiabatic rise of the $40 \mathrm{MHz}$ cavity voltage the bunch length should be around 15 ns. Finally a bunch rotation and compression on harmonic 84 and 168 reduces the bunch length below 4 ns [2] (See Fig. 1)

Table 1: Cavities in use and spare for the $26 \mathrm{GeV} / \mathrm{c}$ flat top RF manipulations

\begin{tabular}{|l|l|l|}
\hline Splitting & Cavity & Number \\
\hline $\mathbf{H 2 1} \rightarrow \mathbf{H 4 2}$ & $10 \mathrm{MHz} \rightarrow \mathbf{2 0} \mathbf{M H z}$. & 1 used, 1 spare \\
\hline $\mathbf{H 4 2} \rightarrow \mathbf{H 8 4}$ & $20 \mathrm{MHz} \rightarrow \mathbf{4 0} \mathbf{M H z}$. & 1 used, 1 spare \\
\hline Bunch rotation & $40 \mathrm{MHz}+\mathbf{8 0} \mathbf{M H z}$. & 2 used, 1 spare \\
\hline
\end{tabular}

For these RF manipulations different types and numbers of cavities are used (see Table 1). For each type of cavity there is a hot spare.

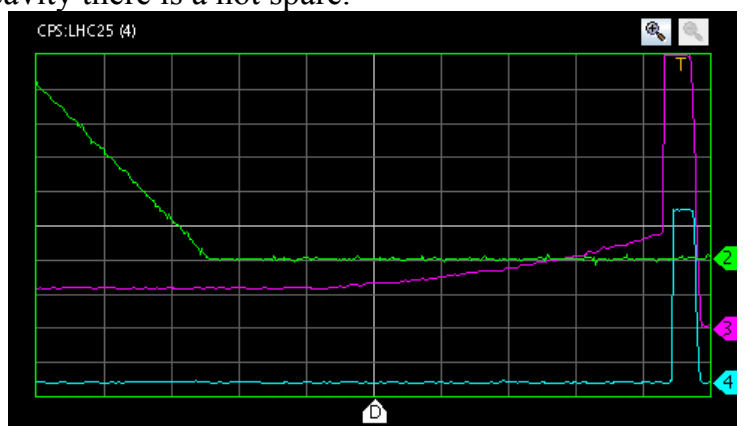

Figure 1: Last part of Flat top RF manipulations, $20 \mathrm{MHz}$ (2), $40 \mathrm{MHz}$ (3), $80 \mathrm{MHz}$ (4) cavity voltages.

\section{INSTABILITY OBSERVATIONS 2006}

During the 2006 SPS scrubbing run the beam was observed to be unstable in the horizontal plane a few milliseconds before the extraction from PS [3]. The tail of the bunch train oscillates horizontally as can be seen on the BPM signal in the PS ring (see Fig. 2) and the BPM signal in the PS to SPS transfer line (see Fig 3). The PS BPM is located inside a combined function magnet.

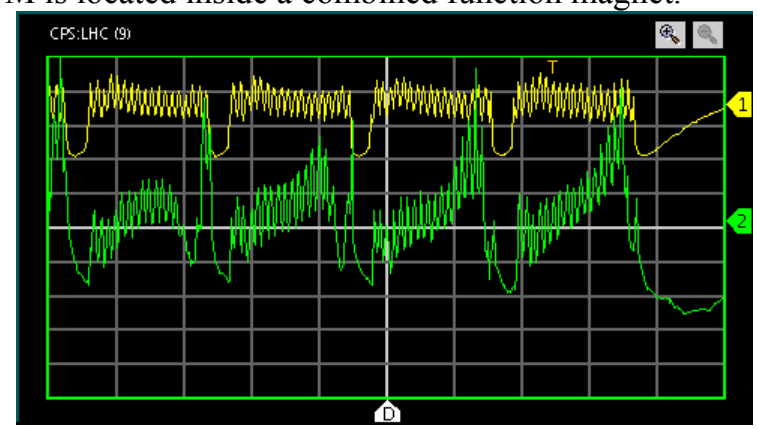

Figure 2: PS ring BPM signals, sum (1) and horizontal delta (2) during the last 4 turns prior to extraction.

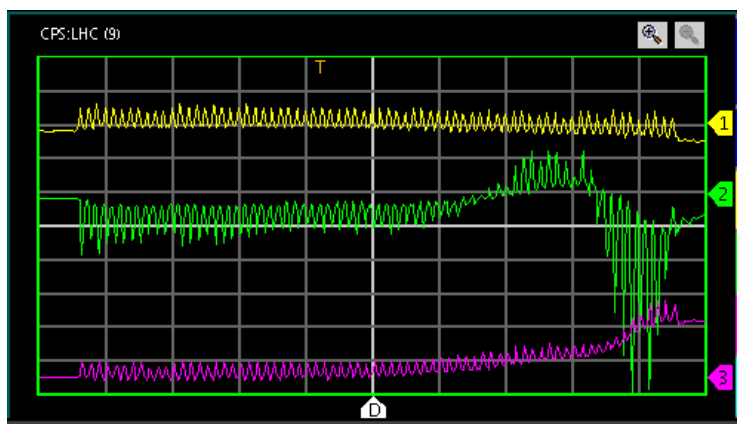

Figure 3: PS to SPS transfer line BPM signals, sum (1), horizontal delta (2) and vertical delta (3). 
Fig. 4 shows the horizontal delta signal of the transverse damper pick-up when connected to a spectrum analyzer operated in receiver mode, tracking the evolution of the $1^{\text {st }}$ spectral betatron line during the last few milliseconds of the cycle. The instability develops very quickly during the adiabatic rise of the $40 \mathrm{MHz}$ cavity, before the bunch rotation, with a rise time of about $1 \mathrm{~ms}$.

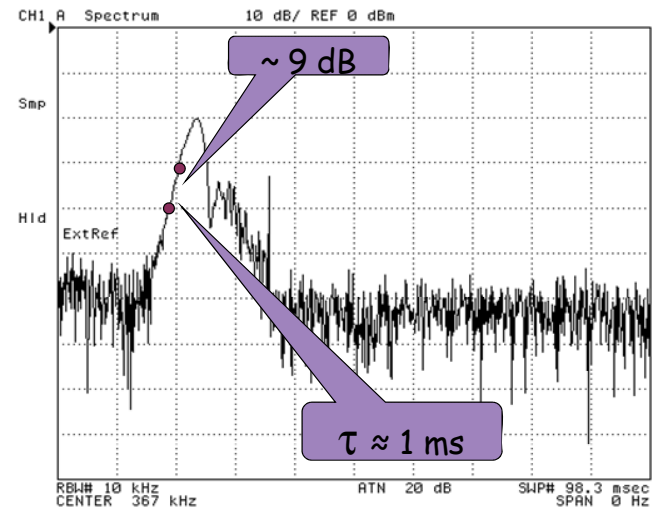

Figure 4: $1^{\text {st }}$ horizontal betatron spectral line in receiver mode during flat top RF manipulations.

The horizontal beam size increases considerably in the case of the instability as can be observed on the fast wire scanners during the last few milliseconds on the extraction flat top (see Fig. 5).
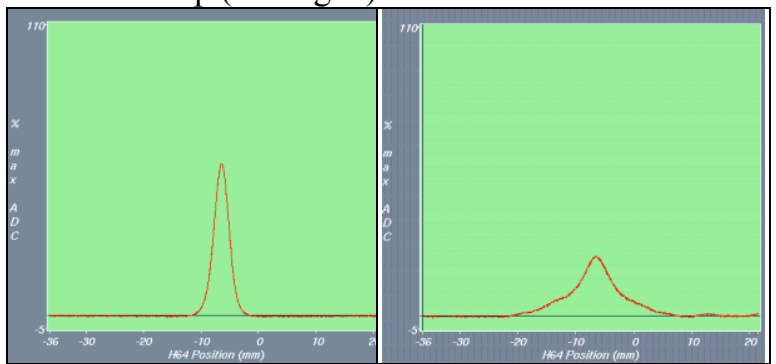

Figure 5: Fast wire scanner profile at extraction in PS, stable (left), unstable (right).

A second instability regime in both transverse planes was identified during the bunch rotation and compression. It has a very fast rise time of about 50 microseconds and should be studied in more detail in the near future.

\section{$40 \mathrm{MHz}$ Cavity Calibration and Bunch Length}

During a machine study session it was discovered that the beam was unstable when using one of the $40 \mathrm{MHz}$ cavities, while the beam was stable when using the other. A measurement campaign to understand the reason for the different behaviour was launched. Measurements were made using both $40 \mathrm{MHz}$ cavities, but one at a time. The bunch rotation process was suppressed and the $40 \mathrm{MHz}$ cavity voltage was blocked at $100 \mathrm{KV}$, which is the nominal voltage prior to the non-adiabatic voltage jump. The full bunch length of 5 bunches along the bunch train was measured for each $40 \mathrm{MHz}$ cavity and resulted in a systematic difference of $1.5 \mathrm{~ns}$ (see Fig. 6).

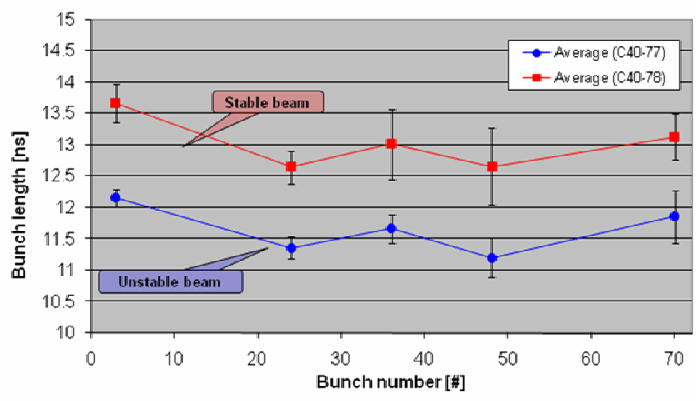

Figure 6: Bunch length difference for the two $40 \mathrm{MHz}$ cavities at $100 \mathrm{KV}$, before calibration $(80 \mathrm{MHz}$ at $0 \mathrm{KV})$.

The 40 and $80 \mathrm{MHz}$ cavities are equipped with a mechanical short circuit which is closed when the cavities are not used while an active feedback is compensating the beam-induced voltage when the cavities are operated. The longitudinal emittance (see Fig. 7) and the bunch length (see Fig. 8) prior to the flat top RF gymnastics were measured with the $40 \mathrm{MHz}$ and $80 \mathrm{MHz}$ cavity gaps open at 0 voltage. No significant difference was observed indicating that the two $40 \mathrm{MHz}$ cavities were not calibrated in the same way and that at least one delivered a different voltage than programmed. This also proved that the active feedback was working properly.

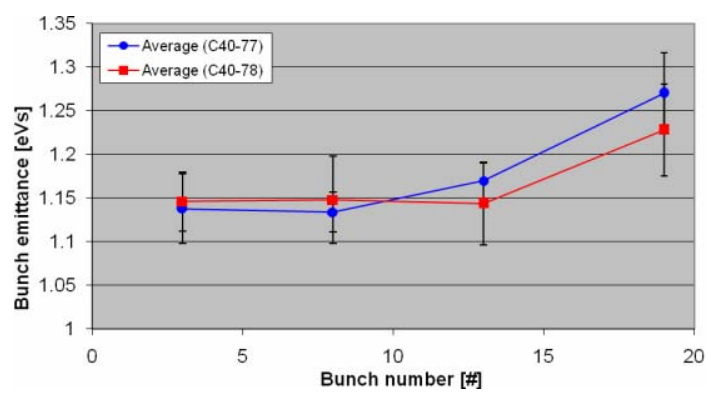

Figure 7: Longitudinal bunch emittance vs. bunch number for each $40 \mathrm{MHz}$ cavity, with gap open and 0 voltage.

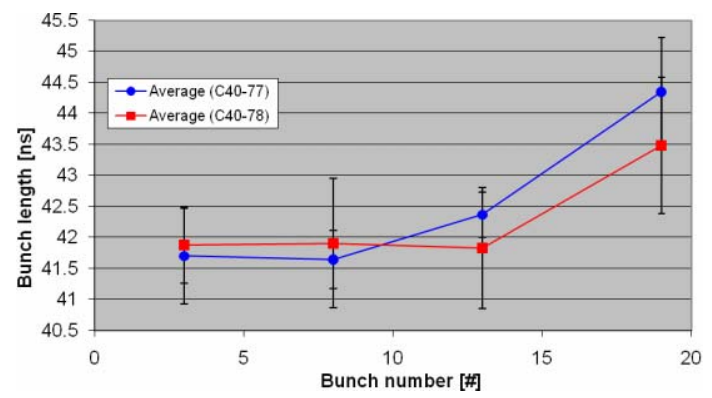

Figure 8: Bunch length vs. bunch number for each 40 $\mathrm{MHz}$ cavity, with gap open and 0 voltage.

The discrepancy in calibration was confirmed and the cavities were re-calibrated. The measurements that followed showed that the bunch length difference before rotation was reduced to $0.5 \mathrm{~ns}$ (see fig. 9). 


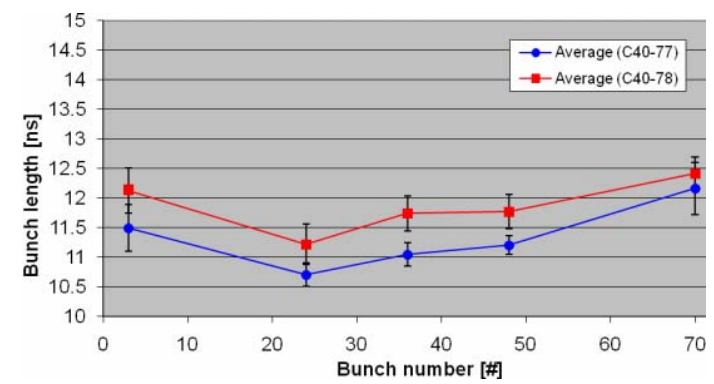

Figure 9: Bunch length difference for the two $40 \mathrm{MHz}$ at $100 \mathrm{KV}$, after calibration ( $80 \mathrm{MHz}$ at $0 \mathrm{KV})$.

The final result of the calibration is that for both cavities the beam was on the edge of instability. A bunch length of $\sim 11.5 \mathrm{~ns}$ seems to be the threshold for the observed instability for the nominal bunch intensity and a longitudinal emittance of $0.35 \mathrm{eVs}$.

\section{COMPARING 2006 WITH 2001}

In 2001 an electron cloud build-up was observed in the PS during the last part of the flat top and in the PS to SPS transfer line, but did not cause the beam to become unstable [4]. A study was launched for which the bunch length was reduced from $\sim 15 \mathrm{~ns}$ to $\sim 10 \mathrm{~ns}$ over an extended flat top of $100 \mathrm{~ms}$. The $1^{\text {st }}$ horizontal spectral betatron line was measured in receiver mode for different intensities and the observed time constant for the development of the instability was $\sim 4$ ms (see Fig. 10).

The main difference compared to the 2006 situation is that the bunch length at the end of the $40 \mathrm{MHz}$ adiabatic voltage rise in 2001 was $\sim 15 \mathrm{~ns}$ and in 2006 it was $\sim 11$ to $\sim 12 \mathrm{~ns}$, which was required in order to provide a clean bunch rotation and therefore minimizing capture losses in the SPS.

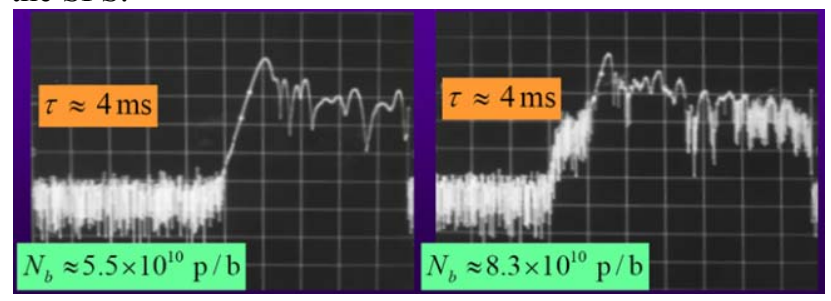

Figure 10: $1^{\text {st }}$ horizontal betatron spectral line in receiver mode during flat top RF manipulations in 2001 for different intensities.

\section{POSSIBLE CURES}

\section{Short Term Solution}

In order to provide stable beam to the SPS for the scrubbing run and the beam tests and to minimize the capture losses in the SPS, a bunch length of $12 \mathrm{~ns}$ at the end of the $40 \mathrm{MHz}$ adiabatic rise seems to be the best compromise.

\section{Longer Term Solution.}

In the long term the aim is to reduce the capture losses in the SPS, by having shorter bunches prior to the bunch rotation, which will reduce the filaments that causes tails in the longitudinal plane at injection in the SPS. A new scheme to do the RF gymnastics has been proposed and should be studied in more detail and tested [5]. This method is based on multiple, well timed non-adiabatic steps, which will generate short bunches in a time shorter than the $1 \mathrm{~ms}$ time constant during which the instability develops.

In parallel electron cloud measurements and simulations, based on the PS parameters, are being performed to better characterize the instability. In addition investigations are ongoing to verify the feasibility of a scrubbing run in the PS to reduce electron multipacting. Furthermore the possibility of using the transverse feedback to damp the observed instabilities is being investigated.

\section{CONCLUSION}

The bunch length threshold in the PS for the instability at nominal intensity and $25 \mathrm{~ns}$ bunch spacing is $11.5 \mathrm{~ns}$. The time constant for the instability is approximately $1 \mathrm{~ms}$ and is very similar to the electron cloud instability observed in 2001. The bunches produced at the end of the $40 \mathrm{MHz}$ adiabatic voltage rise during 2006 were $\sim 4 \mathrm{~ns}$ shorter than in previous years, which is the reason for the reduction in the instability rise-time.

By lengthening the bunches from $\sim 11 \mathrm{~ns}$ to $\sim 12.5 \mathrm{~ns}$ the PS is able to deliver stable nominal LHC beam to the SPS. Additional studies are ongoing to better characterize the instability and identify possible countermeasures (new scheme for bunch rotation, scrubbing and active damping with the transverse feedback) in order to reliably provide the beam to the SPS with smaller bunch length to minimize capture losses.

\section{REFERENCES}

[1] R. Cappi, "Electron Cloud Effects in the PS", PAC'01, Chicago, p 682.

[2] R. Garoby, "LHC Proton Beams in the PS: Status of Preparation and Capabilities", LHC Performance Workshop - Chamonix, CERN-AB-2003-008-ADM.

[3] R. Steerenberg, "Observations of the High Energy Instability in the PS", Accelerator Performance Committee 15-Dec-2006, CERN, Geneva (http://abdiv.web.cern.ch/abdiv/Meetings/APC/2006/apc061215/RS-APC-15-122006.pdf)

[4] R. Cappi et al., "Electron cloud build-up and related instability in the CERN Proton synchrotron." PRSTAB, Volume, 094401 (2002).

[5] H. Damerau, "RF Gymnastics in the PS with the 40 and $80 \mathrm{MHz}$ Cavities", Accelerator Performance Committee 15-Dec-2006, CERN, Geneva (http://abdiv.web.cern.ch/abdiv/Meetings/APC/2006/apc061215/HD-APC-15-122006.ppt) 